\title{
Extraction of the Muon Revolution Frequency Distribution via the Fourier Analysis of the Fast Rotation Signal in the Muon g-2 Experiment
}

Daniel Seleznev ${ }^{1}$

Department of Physics, Cornell University, Ithaca, NY 14850

Science Undergraduate Laboratory Internship

(Dated: 14 August 2017)

The Muon g-2 experiment at Fermi National Accelerator Laboratory aims to measure the anomalous magnetic moment $a_{\mu}$ of the muon to an unprecedented precision of $0.14 \mathrm{ppm}$, obtaining a near four-fold increase in precision over the previous experiment at Brookhaven National Laboratory (BNL). The value of $a_{\mu}$ from BNL differs from the Standard Model prediction by 3.4 standard deviations, suggesting a tantalizing hint of new physics and so motivating a new measurement.

The Fermilab experiment follows the measurement principle from the BNL experiment. A beam of positive muons is stored in the ring, where a combination of magnetic and electric fields helps to focus the beam. The combination of these fields and the stored beam properties directly impact the final extraction of of $a_{\mu}$. The muon anomaly relies on the measurement of the spin precession frequency $\omega_{a}$ about the momentum of the muon. Due to a muon beam momentum spread of approximately $0.1 \%, \omega_{a}$ must be corrected for the effect of a radial electric field. The correction is estimated by means of a modified Fourier analysis of the so-called fast rotation signal. We explore the application of this analysis to the case of a beam with longitudinal spread and to the case of late-time beam detection. We also consider the numerical extension of the method, and apply it to data from the recent commissioning run.

This manuscript has been authored by Fermi Research Alliance, LLC under Contract No. DE-AC02-07CH11359 with the U.S. Department of Energy, Office of Science, Office of High Energy Physics. 


\section{INTRODUCTION}

In the presence of an external magnetic field, fermions gain a magnetic dipole moment

$$
\vec{\mu}=g \frac{Q}{2 m} \vec{s}
$$

where $Q, g$ and $\vec{s}$ are the fermion's electric charge, gyromagnetic ratio, and intrinsic spin vector respectively. One of the great successes of quantum theory was the match between the theoretical and experimental values of $g$ for the electron. Experiments done by the likes of Stern and Gerlach revealed that $g_{e}=2$; this value was later confirmed by Dirac's relativistic theory of the electron. Given the success of Dirac's theory in predicting $g_{e}$, it was anticipated that the $g$-factor of the proton would be 2 as well. However, later findings revealed that the $g$-factor of the proton was $\sim 5.5$, and that the neutron, a particle with no electric charge, also had a high $g$-factor ${ }^{1}$.

With the discovery of different $g$-factors, it became convenient to express them through their deviations from the Dirac value of $g=2: g=2(1+a)$, where the first piece is the Dirac moment and the second piece, $a$, is the deviation. Theoretically, the deviations were found to be the result of radiative corrections to the Dirac moment. For the muon, $a_{\mu}$ results from all the particles that couple to the muon, so a precise determination of the muon anomaly can serve as a gateway to the discovery of new physics. Additionally, the fact that $a_{\mu}$ can be found to a high degree of precision from theory and experiment makes it of particular usefulness for such ventures ${ }^{1}$. The current predicted and experimental values of $a_{\mu}$ are determined to a precision of $0.5 \mathrm{ppm}$. However, there is a discrepancy of $\sim 3.4 \sigma$ between these two values. To explain this discrepancy, Standard Model extensions such as extra dimensions, supersymmetry, and dark matter candidates have been proposed. But due to the fact that the BNL experiment was statistics limited, the discrepancy is possibly just a statistical fluctuation ${ }^{2}$. The discrepancy must be resolved, thus justifying a new Muon g-2 experiment.

The Fermilab E989 Muon g-2 experiment aims to measure $a_{\mu}$ to a precision of $0.14 \mathrm{ppm}$, a near four-fold increase over the previous measurement, and so hopes to provide an answer to the questions outlined above. The experiment relies on the storage of muons inside a weak focusing ring, with a continuous C-shape dipole magnet occupying its entirety (44.7 $\mathrm{m}$ circumference). The dipole generates a vertical and highly homogenous $1.45 \mathrm{~T}$ magnetic field to provide radial focusing for muon storage. The field intensity corresponds to storing 
muons with a design momentum of $3.094 \mathrm{GeV} / c$ onto the design orbit (7.112 m radius), on which the muons undergo a cyclotron motion with a revolution frequency of 149.1 ns. Vertical focusing is provided by four electrostatic quadrupoles (ESQs) located around the ring.

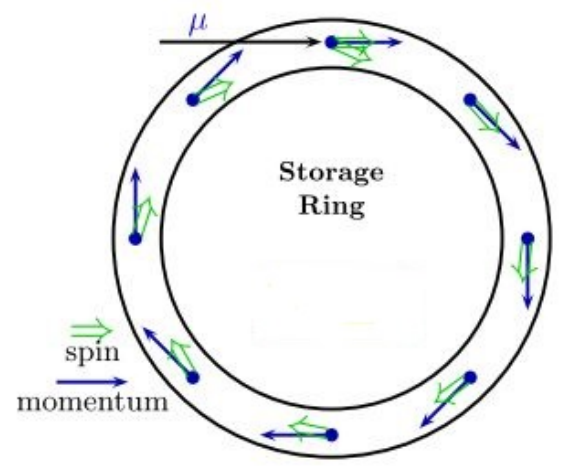

FIG. 1. Precession of a muon's spin about its momentum on the design orbit ${ }^{3}$

The final extraction of $a_{\mu}$ is performed via the measurement of the intensity of the magnetic field in term of the Larmor precession frequency of a free proton

$$
\hbar \omega_{p}=2 \mu_{p}|\vec{B}|
$$

and the precession frequency $\omega_{a}$ of the muon's spin about its momentum. $\omega_{a}$ is obtained by subtracting the cyclotron frequency $\omega_{C}$ from the total spin precession frequency of the $\operatorname{muon}^{2} \omega_{S}$ :

$$
\omega_{a}=\omega_{S}-\omega_{C}
$$

We see a schematic representation of the muon spin precession in Figure 1. In the presence of the electric field, $\omega_{a}$ is given $b^{2}$

$$
\vec{\omega}_{a}=-\frac{Q}{m}\left[a_{\mu} \vec{B}-\left(a_{\mu}-\frac{1}{\gamma^{2}-1}\right) \frac{\vec{\beta} \times \vec{E}}{c}\right]
$$

The term proportional to $\vec{\beta} \times \vec{E}$ in Eq. (4) corresponds to the electric field contribution to $\omega_{a}$. One can see that if $a_{\mu}=\frac{1}{\gamma^{2}-1}$ the contribution disappears. This is the approach followed by the previous CERN and BNL E821 experiments. The magic momentum of $3.094 \mathrm{GeV} / \mathrm{c}$ allows the electric field contribution to vanish in first order. The correction for the electric field contribution to $\omega_{a}$ is obtained from the muon revolution frequency distribution which is 
the result of a modified Fourier analysis of the so-called fast rotation signal (FRS). The FRS describes the beam intensity as seen by a detector at a fixed location in the ring assuming stable particles. The technique was created for $\mathrm{E} 821^{4}$, but we independently re-derive its following key aspects: the analysis for when the beam features a longitudinal spread, and the analysis if beam detection occurs at late times. We also consider the numerical extension of the method, and apply it to data from the recent commissioning run.

\section{BACKGROUND}

\section{A. Mathematical Background}

Definition 1 (Fourier's transform and inverse transform) Given a function $f$, its Fourier transform is

$$
\hat{f}(x)=\frac{1}{\sqrt{2 \pi}} \int_{-\infty}^{\infty} f(t) e^{i x t} d t
$$

and its inverse Fourier transform is

$$
\tilde{f}(x)=\frac{1}{\sqrt{2 \pi}} \int_{-\infty}^{\infty} f(t) e^{-i x t} d t
$$

If $f$ is absolutely integrable, then the Fourier transforms of $f$ always exist. It is easy to check that $f=\tilde{\hat{f}}=\hat{\tilde{f}}$.

Theorem 1 (Fourier's Integral ${ }^{5}$ ) For any piecewise continuous, piecewise differentiable, and absolutely integrable function $f$, the following equality holds:

$$
f(x)=\frac{1}{\pi} \int_{0}^{\infty} d \omega \int_{-\infty}^{\infty} f(t) \cos \omega(t-x) d t
$$

If $f$ is even, Fourier's integral simplifies to

$$
f(x)=\frac{2}{\pi} \int_{0}^{\infty} \cos \omega x d \omega \int_{0}^{\infty} f(t) \cos \omega t d t
$$

Suppose $f$ is a piecewise continuous, piecewise differentiable, and absolutely integrable function on $[0, \infty)$. If we extend $f$ to an even function defined on the whole real line, then the formula above is valid for the extension. In particular, it is also valid for $x \geq 0$.

Theorem 2 (Convolution Theorem) The convolution of two functions $f$ and $g$ is defined to be

$$
(f * g)(t)=\frac{1}{\sqrt{2 \pi}} \int_{-\infty}^{\infty} f(\tau) g(t-\tau) d \tau
$$


The convolution theorem states that

$$
\widehat{f * g}=\hat{f} \hat{g} \text { and } \widetilde{f * g}=\tilde{f} \tilde{g}
$$

Definition 2 (Discrete Fourier Transform) Given a function $f$ acting on set sample of points $\left\{x_{0}, x_{1}, \ldots, x_{N-1}\right\}$, the discrete Fourier transform (DFT) of $f$ is a set of points $\left\{s_{0}, s_{1}, \ldots, s_{N-1}\right\}$ given by

$$
s_{m}=\sum_{n=0}^{N-1} f_{n} e^{2 \pi i m n / N}
$$

where $f_{n}=f\left(x_{n}\right)$. The DFT is the discrete analog of the Fourier transform.

\section{B. Physical Background}

Given a beam with a momentum spread $\rho(\Delta)$ and no longitudinal spread or transverse emittance, the FRS of the beam at a detector with azimuthal position $\theta$ and no azimuthal width is given by

$$
S_{0}(t)=\sum_{n=0}^{\infty} \frac{\rho\left(\frac{t}{(n+\theta / 2 \pi) T}-1\right)}{(n+\theta / 2 \pi) T}
$$

where $n$ is the turn number and $T$ the cyclotron period of a particle on the design orbit. The muon beam is expected to have a narrow spread in revolution frequencies, $\left(\omega-\omega_{0}\right) / \omega \sim 10^{-3}$ where $\omega_{0}$ is the design orbit frequency, due to the aperture size of the storage region. As a result the beam will spread negligibly in the longitudinal direction during the first turn. We thus have that

$$
\frac{\theta}{2 \pi}=\frac{t_{0}}{T}
$$

where $t_{0}$ is the time the center of mass of the beam first passes the detector. We may thus rewrite the FRS above as

$$
S_{0}(t)=\sum_{n=0}^{\infty} \frac{\rho\left(\frac{t}{n T+t_{0}}-1\right)}{n T+t_{0}}
$$

We see an example of an FRS in Figure 2. 


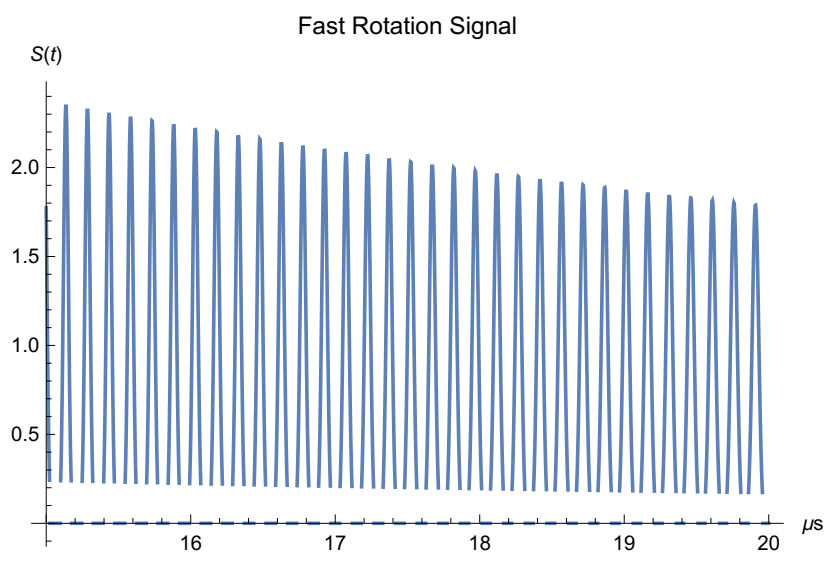

FIG. 2. FRS for a beam with a Gaussian momentum distribution with a spread of $0.112 \%$ and no longitudinal spread or transverse emittance, as seen by a detector at a point halfway around the storage ring. The beam is observed to debunch with the increasing width of consecutive peaks.

$S_{0}(t)$ is time reversal symmetric about $t_{0}$; therefore its Fourier transform is given by

$$
\hat{S}_{0}(\omega)=\sqrt{\frac{2}{\pi}} \int_{t_{0}}^{\infty} S_{0}(t) \cos \omega\left(t-t_{0}\right) d t
$$

where infinite time represents a time after the beam has fully decohered. We may obtain $S_{0}(t)$ from $\hat{S}_{0}(\omega)$ through the Fourier transform

$$
S_{0}(t)=\sqrt{\frac{2}{\pi}} \int_{0}^{\infty} \hat{S}_{0}(\omega) \cos \omega\left(t-t_{0}\right) d \omega
$$

Figure 3 features a comparison of the first harmonic of $\hat{S}_{0}(\omega)$ to the actual revolution frequency distribution of the muons for the FRS of Figure 2. We see they match exactly.

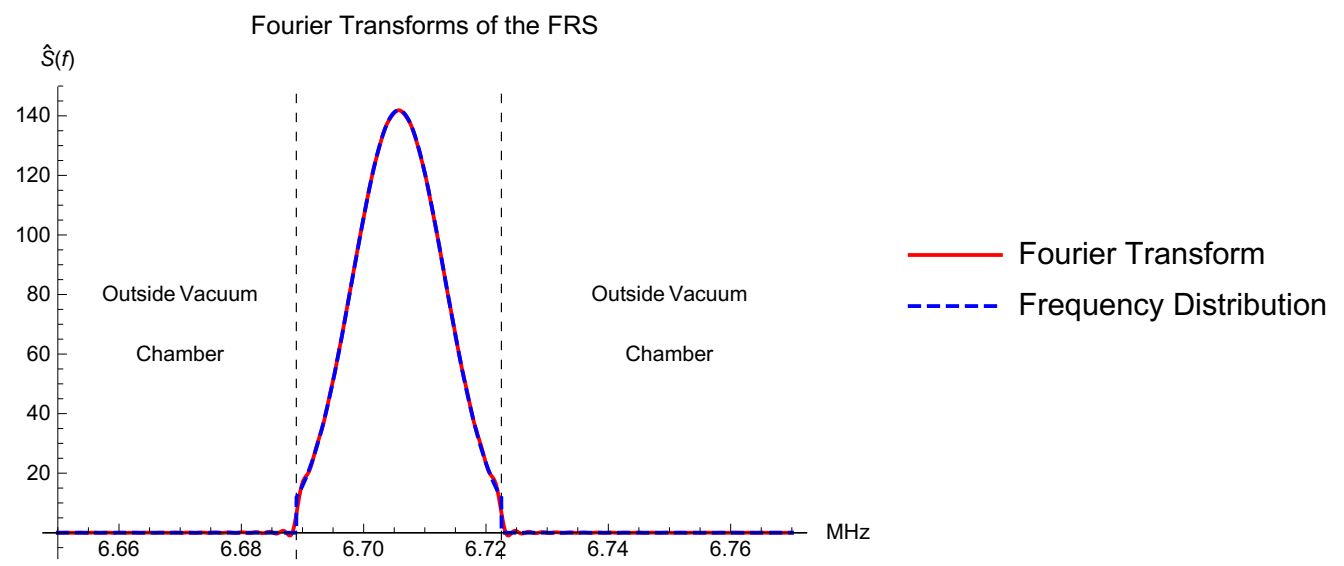

FIG. 3. Comparison of the first harmonic of the Fourier transform of the FRS from Figure 2 to the actual muon revolution frequency distribution 
It makes sense to extract the frequency distribution of the muons from the FRS because the signal arises from the periodic detector hits of the muons. The periods of these hits depend on the revolution frequencies that the muons have, so the Fourier transform of the FRS reflects the relative amounts of these frequencies among the muons. The Fourier transform will feature peaks centered at integer multiples of the design frequency $\omega_{0}$. However, the muons are restricted to the vacuum chamber. The frequencies corresponding to the exterior of the chamber are an artifact of the Fourier transform. Hence the first harmonic corresponds to the actual revolution frequency distribution of the muons.

\section{ANALYSIS FOR A BEAM WITH LONGITUDINAL SPREAD}

\section{A. The Case of Symmetric Longitudinal Spread}

Assuming that there is no initial correlation between muon revolution frequencies and longitudinal phases, there will exist a longitudinal offset distribution $\xi\left(t^{\prime}\right)$ which must be taken into account when performing the Fourier analysis. The FRS for a beam with a longitudinal spread is given by

$$
S(t)=\int \xi\left(t^{\prime}\right) S_{0}\left(t-t^{\prime}\right) d t^{\prime}=\sqrt{2 \pi}\left(\xi * S_{0}\right)(t)
$$

An example of such a FRS is given in Figure 4.

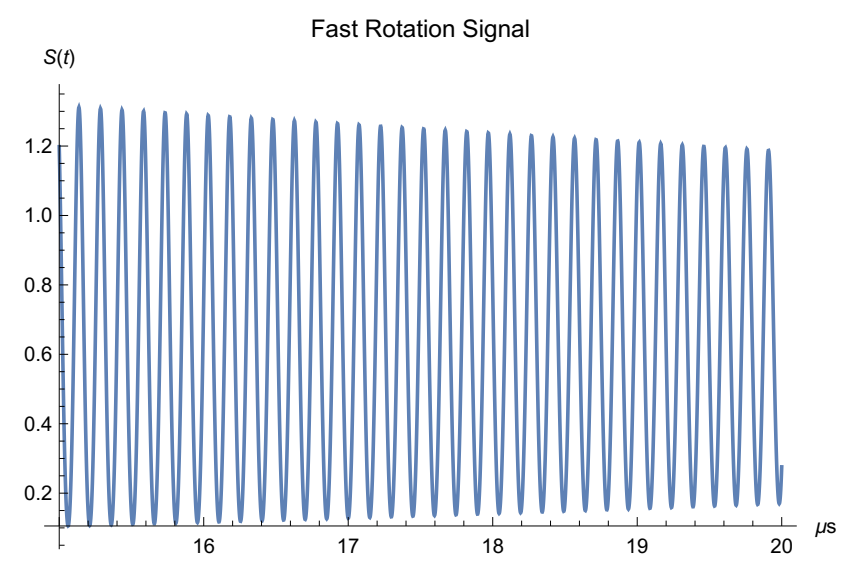

FIG. 4. FRS for a beam with Gaussian momentum distribution of spread $0.112 \%$ and with a Gaussian longitudinal distribution with $\sigma_{t}=25 \mathrm{~ns}$, as seen by a detector at a point halfway around the storage ring. 
Ideally, the application of the convolution theorem would yield

$$
\hat{S}(\omega)=\sqrt{2 \pi} \hat{\xi}(\omega) \hat{S}_{0}(\omega)
$$

However, neither $S_{0}(t)$ nor $\xi(t)$ will be available to work with directly in the experiment, leaving only $S(t)$ to get $\hat{S}(\omega)$.

We express $S_{0}\left(t-t^{\prime}\right)$ through Fourier's integral:

$$
\begin{gathered}
S_{0}\left(t-t^{\prime}\right)=\frac{2}{\pi} \int_{0}^{\infty} \cos \omega\left(t-t_{0}-t^{\prime}\right) d \omega \int_{t_{0}}^{\infty} S_{0}(\bar{t}) \cos \omega\left(\bar{t}-t_{0}\right) d \bar{t}= \\
\sqrt{\frac{2}{\pi}} \int_{0}^{\infty} \hat{S}_{0}(\omega) \cos \omega\left(t-t_{0}-t^{\prime}\right) d \omega
\end{gathered}
$$

Substituting this expression into the convolution integral, we get

$$
\begin{gathered}
S(t)=\sqrt{2 \pi}\left(\xi * S_{0}\right)(t)=\int_{-\infty}^{\infty} \xi(t) S_{0}\left(t-t^{\prime}\right) d t^{\prime}=\sqrt{\frac{2}{\pi}} \int_{0}^{\infty} \hat{S}_{0}(\omega) d \omega \int_{-\infty}^{\infty} \xi\left(t^{\prime}\right) \cos \omega\left(t-t_{0}-t^{\prime}\right) d t^{\prime}= \\
\sqrt{\frac{2}{\pi}} \int_{0}^{\infty} \hat{S}_{0}(\omega)\left\langle\cos \omega\left(t-t_{0}-t^{\prime}\right)\right\rangle d \omega= \\
\sqrt{\frac{2}{\pi}} \int_{0}^{\infty} \hat{S}_{0}(\omega)\left\langle\cos \omega\left(t-t_{0}\right) \cos \omega t^{\prime}+\sin \omega\left(t-t_{0}\right) \sin \omega t^{\prime}\right\rangle d \omega= \\
\sqrt{\frac{2}{\pi}}\left(\int_{0}^{\infty} \hat{S}_{0}(\omega)\left\langle\cos \omega t^{\prime}\right\rangle \cos \omega\left(t-t_{0}\right) d \omega+\int_{0}^{\infty} \hat{S}_{0}(\omega)\left\langle\sin \omega t^{\prime}\right\rangle \sin \omega\left(t-t_{0}\right) d \omega\right)
\end{gathered}
$$

where \langle\rangle denotes averaging with respect to $\xi(t)$.

If $\xi\left(t^{\prime}\right)$ is even, $\left\langle\sin \omega t^{\prime}\right\rangle=0$, leaving

$$
S(t)=\sqrt{\frac{2}{\pi}} \int_{0}^{\infty} \hat{S}_{0}(\omega)\left\langle\cos \omega t^{\prime}\right\rangle \cos \omega\left(t-t_{0}\right) d \omega
$$

suggesting that $\hat{S}(\omega)=\hat{S}_{0}(\omega)\left\langle\cos \omega t^{\prime}\right\rangle$. We can see that this is true by referring back to (9):

$$
\hat{\xi}(\omega)=\frac{1}{\sqrt{2 \pi}} \int_{-\infty}^{\infty} \xi\left(t^{\prime}\right) e^{i \omega t^{\prime}} d t^{\prime}=\frac{1}{\sqrt{2 \pi}} \int_{-\infty}^{\infty} \xi\left(t^{\prime}\right) \cos \omega t^{\prime} d t^{\prime}=\frac{\left\langle\cos \omega t^{\prime}\right\rangle}{\sqrt{2 \pi}}
$$

and therefore

$$
\hat{S}(\omega)=\sqrt{2 \pi} \hat{\xi}(\omega) \hat{S}_{0}(\omega)=\hat{S}_{0}(\omega)\left\langle\cos \omega t^{\prime}\right\rangle
$$

Thus (10) implies that

$$
\hat{S}(\omega)=\sqrt{\frac{2}{\pi}} \int_{t_{0}}^{\infty} S(t) \cos \omega\left(t-t_{0}\right) d t
$$


In Figure 5 we see the first harmonics of the Fourier transforms of an FRS with longitudinal spread from Formulas (9) and (12) compared to the actual frequency distribution. We see that they match.

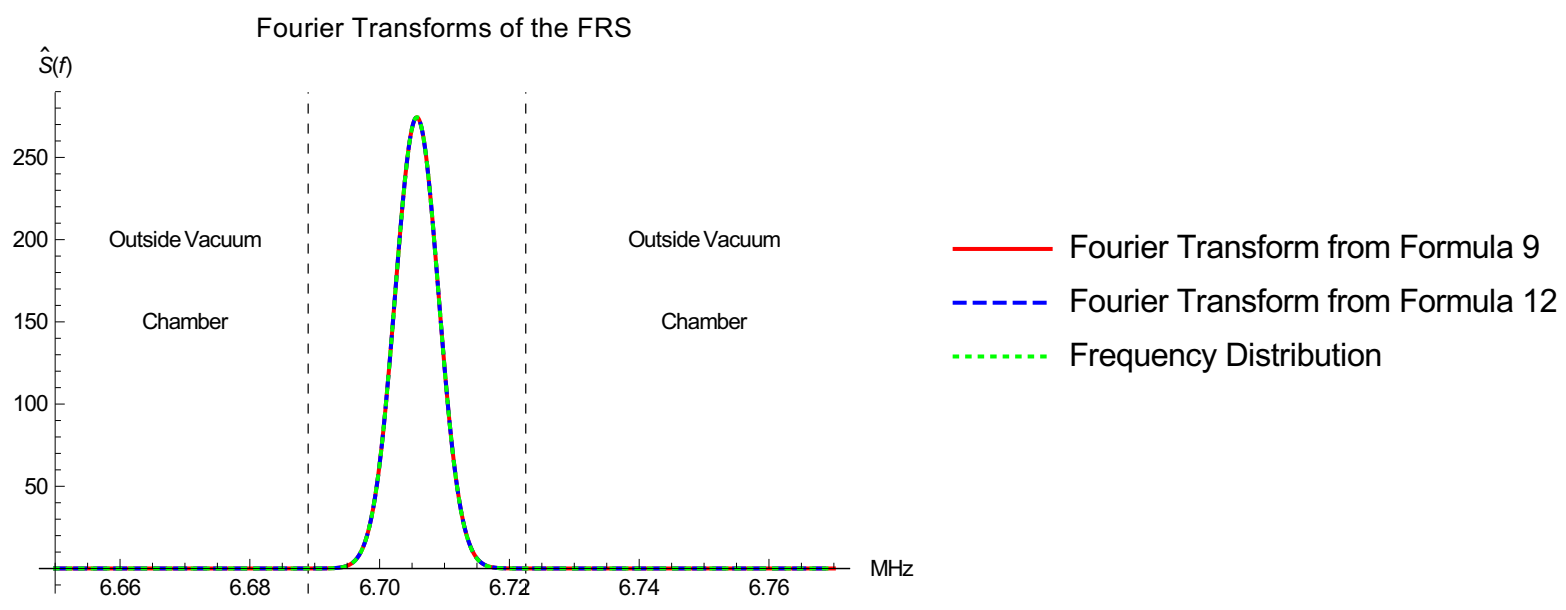

FIG. 5. Comparison of the first harmonics of the Fourier transforms to the frequency distribution for an FRS with Gaussian momentum distribution of spread 0.05\% and a Gaussian longitudinal spread with $\sigma_{t}=10 \mathrm{~ns}$, as seen by a detector at a point halfway around the storage ring. $t_{0}=74.563$ ns.

\section{B. Asymmetric Longitudinal Bunch Distribution}

If $\xi\left(t^{\prime}\right)$ is not symmetric, then $\hat{S}(\omega)$ features an imaginary component. Directly applying the convolution theorem yields $\hat{S}(\omega)=\hat{S}_{0}(\omega)\left\langle\cos \omega t^{\prime}\right\rangle+i \hat{S}_{0}(\omega)\left\langle\sin \omega t^{\prime}\right\rangle$ which results in an imaginary radial electric field correction.

To motivate a solution to this problem, we examine a specific example of an asymmetric longitudinal distribution. Suppose $\xi$ is even about some point $\mu \neq 0$ (also the center of mass of the distribution), that is $\xi(x+\mu)=\xi(\mu-x)$. Let $t_{0}$ be the time that the center of mass of the beam first arrives at a given detector if $\mu$ were to equal 0 . Then the actual arrival time of the center of mass of the bunch at the detector is $t_{0}^{\prime}=t_{0}+\mu$. The way that the beam appears to have evolved is the same as if it had a symmetric bunch profile and a detector at azimuthal position $\theta=2 \pi t_{0}^{\prime} / T$ was used (see Figure 6). Define $\xi^{\prime}(t)=\xi(t+\mu)$ and $S_{0}^{\prime}(t)=S_{0}(t-\mu)$. Then the physical situation described by the triple $\left(S_{0}^{\prime}, \xi^{\prime}, t_{0}^{\prime}\right)$ is the 
same as that described by $\left(S_{0}, \xi, t_{0}\right)$. In fact we may see that they yield the same FRS $S(t)$ :

$$
\begin{gathered}
\sqrt{2 \pi} \xi^{\prime}(t) * S_{0}^{\prime}(t)=\int_{-\infty}^{\infty} \xi^{\prime}(\tau) S_{0}^{\prime}(t-\tau) d \tau=\int_{-\infty}^{\infty} \xi(\tau+\mu) S_{0}(t-\tau-\mu) d \tau=\int_{-\infty}^{\infty} \xi\left(\tau^{\prime}\right) S_{0}\left(t-\tau^{\prime}\right) d \tau^{\prime}= \\
\sqrt{2 \pi} \xi(t) * S_{0}(t)=S(t)
\end{gathered}
$$

where we used the change of variables $\tau^{\prime}=\tau+\mu$. But if we use $\left(S_{0}^{\prime}, \xi^{\prime}, t_{0}^{\prime}\right)$ for the Fourier analysis, the imaginary term in $\hat{S}(\omega)$ vanishes, since we're now dealing with a familiar situation: a symmetric longitudinal distribution. Therefore

$$
\hat{S}(\omega)=\sqrt{\frac{2}{\pi}} \int_{t_{0}^{\prime}}^{\infty} S(t) \cos \omega\left(t-t_{0}^{\prime}\right) d t
$$

What was done mathematically is the following. A function

$$
f(x)=\int_{\xi_{--x}}^{\xi_{+}-x} \xi(t+x) \sin \omega t d t \approx \int_{\xi_{--x}}^{\xi_{+}-x} \xi(t+x) \sin \omega_{0} t d t
$$

was defined. The approximation is made because we are concerned with extracting the first harmonic of the Fourier transform, where the frequency distribution is narrow and centered around $\omega_{0} \cdot\left(\xi_{-}, \xi_{+}\right)$is the domain of $\xi(t)$, and a point $\mu$ such that $f(\mu)=0$ is then found. This effectively amounts to finding a new origin about which $\left\langle\sin \omega t^{\prime}\right\rangle=0$, so that there is no imaginary component to the frequency distribution computed from the Fourier transform.

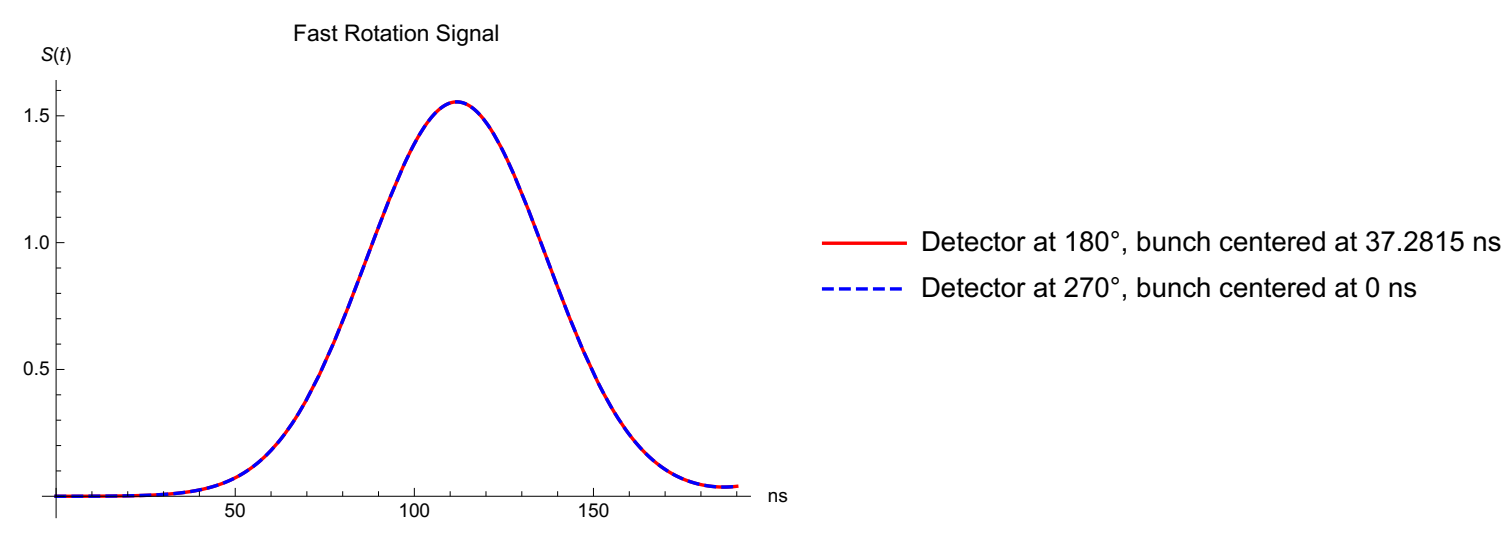

FIG. 6. Both beams have Gaussian momentum distribution with a spread of $0.112 \%$ and Gaussian longitudinal spread with $\sigma_{t}=25 \mathrm{~ns}$. The detector halfway around the ring is witnessing a beam with an offset Gaussian bunch, while the second is detecting one centered at the origin.

When $\xi(t)$ is an arbitrary asymmetric distribution, there is generally no unique symmetry point which makes it obvious how to redefine $t_{0}$. However, we can redefine $t_{0}$ to be $t_{0}^{\prime}=t_{0}+x_{0}$ 
where $x_{0}$ is a root of $f(x)$. Once $t_{0}^{\prime}$ is found, it is used instead of $t_{0}$ in the Fourier integrals. In Figures 7 and 8 we see an example of a FRS with asymmetric bunch profile and the first harmonic of its Fourier transform compared to the actual revolution frequency distribution.

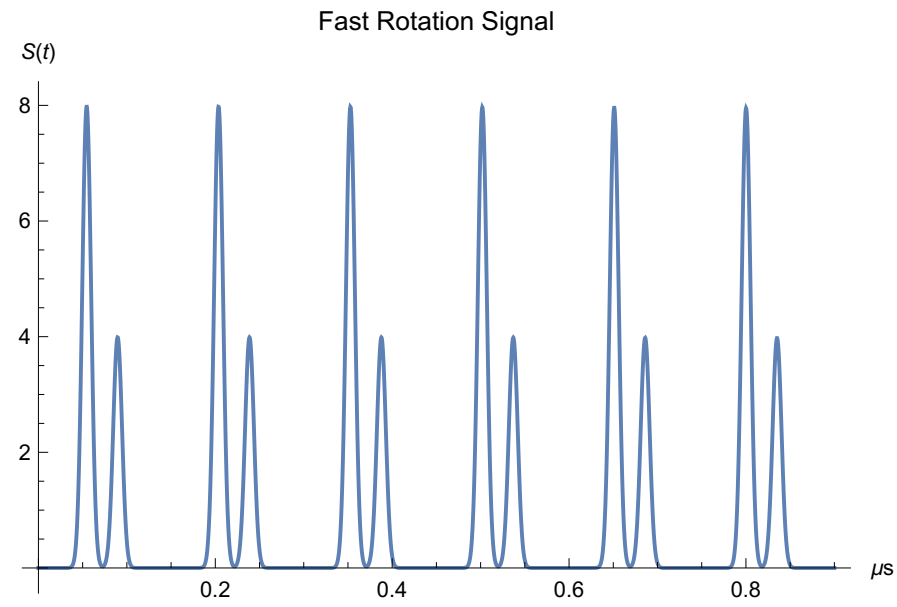

FIG. 7. FRS for a beam with asymmetric longitudinal bunch profile seen by a detector halfway around the ring.

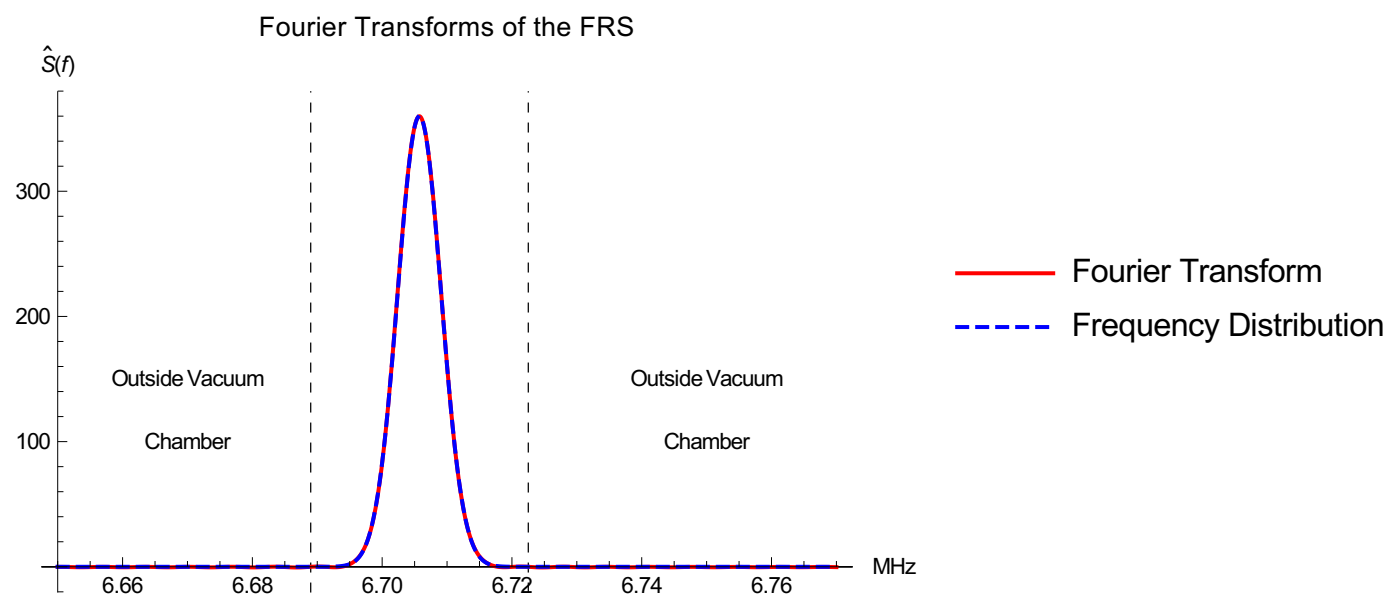

FIG. 8. Comparison of the first harmonic of the Fourier transform of the FRS from Figure 7 to the actual muon revolution frequency distribution. The Fourier analysis used $t_{0}=65.086 \mathrm{~ns}$.

\section{ANALYSIS FOR LATE DETECTION OF THE BEAM}

If the detector detects the muons at a time $t_{s}>t_{0}$, a fast rotation signal $S_{1}(t)$ which vanishes on $\left(t_{0}, t_{s}\right)$ and equals the full fast rotation signal $S(t)$ on $\left(t_{s}, \infty\right)$ is observed. Taking the Fourier transform of $S_{1}(t)$, results in the frequency spectrum 


$$
\hat{S}_{1}(\omega)=\sqrt{\frac{2}{\pi}} \int_{t_{s}}^{\infty} S_{1}(t) \cos \omega\left(t-t_{0}\right) d t
$$

This is not the complete frequency spectrum as the following component is missing:

$$
\Delta(\omega)=\sqrt{\frac{2}{\pi}} \int_{t_{0}}^{t_{s}} S(t) \cos \omega\left(t-t_{0}\right) d t
$$

The problem is to obtain an approximation for $\Delta(\omega)$ using only $S_{1}(t)$.

We first obtain an expression for $\Delta(\omega)$ using $\hat{S}(\omega)$ :

$$
\Delta(\omega)=\sqrt{\frac{2}{\pi}} \int_{t_{0}}^{t_{s}} S(t) \cos \omega\left(t-t_{0}\right) d t=\frac{2}{\pi} \int_{t_{0}}^{t_{s}} \int_{0}^{\infty} \hat{S}\left(\omega^{\prime}\right) \cos \omega^{\prime}\left(t-t_{0}\right) \cos \omega\left(t-t_{0}\right) d \omega^{\prime} d t
$$

Switching the order of integration to integrate with respect to $t$ first, yields

$$
\Delta(\omega)=\frac{1}{\pi} \int_{0}^{\infty} \hat{S}\left(\omega^{\prime}\right)\left(\frac{\sin \left(\omega-\omega^{\prime}\right)\left(t_{s}-t_{0}\right)}{\omega-\omega^{\prime}}+\frac{\sin \left(\omega+\omega^{\prime}\right)\left(t_{s}-t_{0}\right)}{\omega+\omega^{\prime}}\right) d \omega^{\prime}
$$

The vacuum chamber is given by the interval $\left(\omega_{1}^{-}, \omega_{1}^{+}\right)$, with the first harmonic lying in that interval. The $n$-th harmonic then belongs to the interval $\left(\omega_{n}^{-}, \omega_{n}^{+}\right)=\left(\omega_{1}^{-}+(n-1) \omega_{0}, \omega_{1}^{+}+\right.$ $\left.(n-1) \omega_{0}\right)$ where $\omega_{0}$ is the magic frequency. $\hat{S}(\omega)$ is non-zero only on the intervals $\left(\omega_{n}^{-}, \omega_{n}^{+}\right)$, so the above integral may be rewritten as

$$
\sum_{n=1}^{\infty} \frac{1}{\pi} \int_{\omega_{n}^{-}}^{\omega_{n}^{+}} \hat{S}\left(\omega^{\prime}\right)\left(\frac{\sin \left(\omega-\omega^{\prime}\right)\left(t_{s}-t_{0}\right)}{\omega-\omega^{\prime}}+\frac{\sin \left(\omega+\omega^{\prime}\right)\left(t_{s}-t_{0}\right)}{\omega+\omega^{\prime}}\right) d \omega^{\prime}
$$

As such, the second term in the parentheses is neglected, as it is negligibly small:

$$
\Delta(\omega)=\sum_{n=1}^{\infty} \frac{1}{\pi} \int_{\omega_{n}^{-}}^{\omega_{n}^{+}} \hat{S}\left(\omega^{\prime}\right) \frac{\sin \left(\omega-\omega^{\prime}\right)\left(t_{s}-t_{0}\right)}{\omega-\omega^{\prime}} d \omega^{\prime}
$$

The Fourier transform has associated with it an uncertainty principle ${ }^{6}$, namely that $\Delta \omega \Delta t \sim 2 \pi$. The uncertainty principle implies that if $t_{s}-t_{0}$ is sufficiently small, the spread of $\hat{S}_{1}(\omega)$ within $\left(\omega_{n}^{-}, \omega_{n}^{+}\right)$will change negligibly relative to that of $\hat{S}(\omega)$ for all $n$. This means that the frequency content of $\hat{S}_{1}(\omega)$ is approximately the same as that of $\hat{S}(\omega)$. This is evident from Figure 9. 


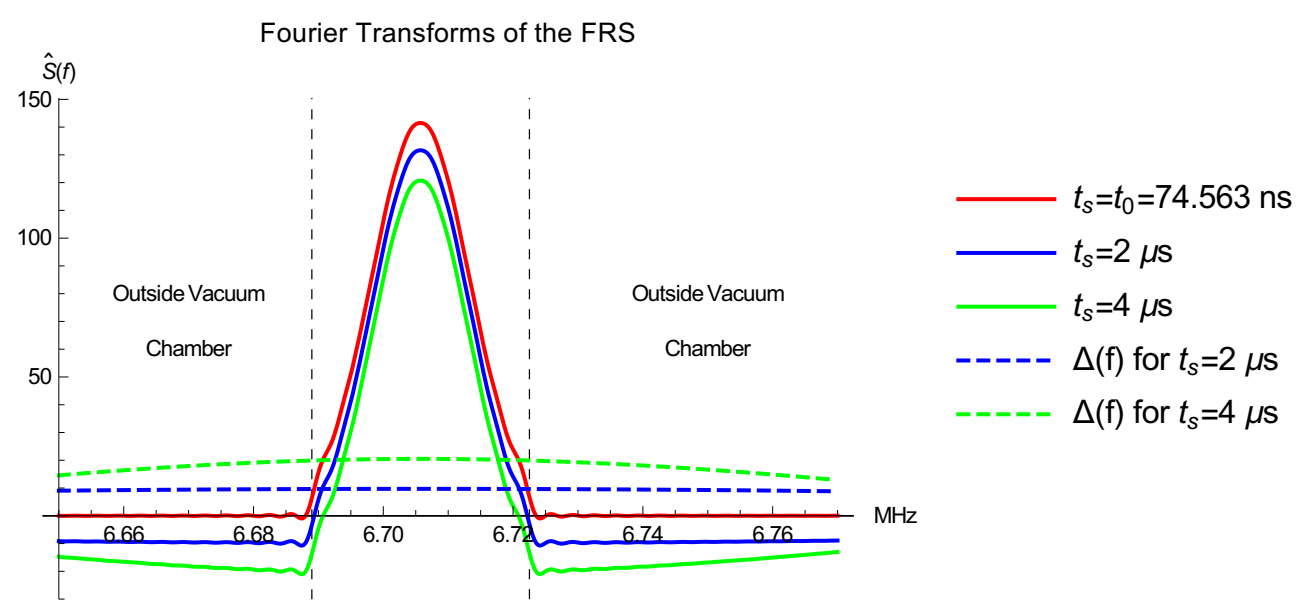

FIG. 9. Frequency spectra using different start times $t_{s}$ for the FRS of Figure 2, as well the corresponding $\Delta(f)$ functions. The spread of $\hat{S}_{1}(\omega)$ is unchanged relative to $\hat{S}(\omega)$.

With $t_{s}>t_{0}, \hat{S}_{1}(\omega)$ features frequencies outside the vacuum chamber boundaries due to aliasing effects. However, these are non-physical as no muons can actually be outside the chamber. Due to the uncertainty principle, the spread of $\hat{S}_{1}(\omega)$ in $\left(\omega_{n}^{-}, \omega_{n}^{+}\right)$remained approximately the same as that of $\hat{S}(\omega)$. And since the only physical frequencies lie within that interval, we approximate $\Delta(\omega)$ by writing

$$
\Delta(\omega) \approx \sum_{n=1}^{\infty} \frac{1}{\pi} \int_{\omega_{n}^{-}}^{\omega_{n}^{+}} \hat{S}_{1}\left(\omega^{\prime}\right) \frac{\sin \left(\omega-\omega^{\prime}\right)\left(t_{s}-t_{0}\right)}{\omega-\omega^{\prime}} d \omega^{\prime}
$$

The Fourier transform of a function measures the relative amounts of sines and cosines of all frequencies in that function. Therefore, the absolute amplitude of the Fourier transform is irrelevant; a way of seeing this is considering the following. Say $\hat{f}(\omega)$ is the Fourier transform of a signal $f(t)$. Let's vertically shift the Fourier transform: $\hat{f} \rightarrow \hat{f}+c$. The inverse Fourier transform of this will be $f(t)+c \delta(t)$, where $\delta(t)$ is the Dirac delta function. However, $\delta(t)$ carries no real physical meaning in this scenario, leaving only the spread of the Fourier transform as carrying significance.

The argument for using this approximation relies on the fact that $\hat{S}_{1}(\omega)$ has a spread comparable to $\hat{S}(\omega)$. So when $\Delta$ is computed from $\hat{S}_{1}(\omega)$, we find that it has spread comparable to the actual $\Delta$. Their absolute amplitudes will differ, but their spreads will be comparable, making this approximation a valid one. 


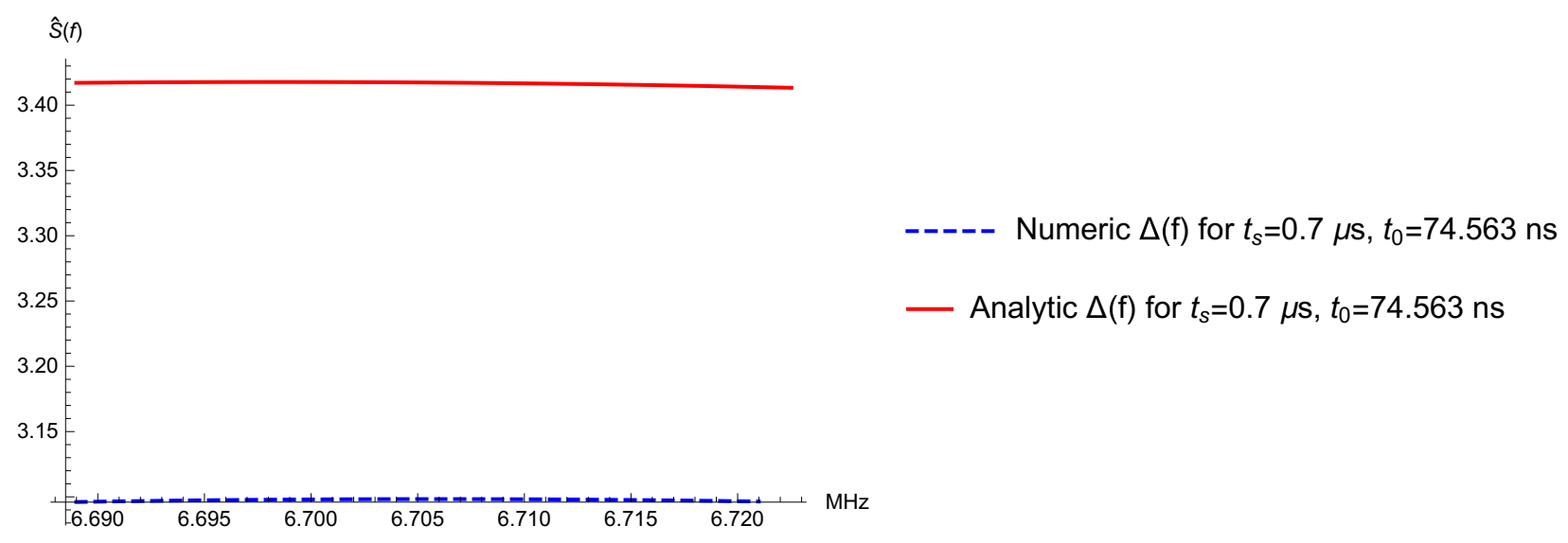

FIG. 10. The actual and approximated $\Delta(f)$ functions for the beam of Figure 2 for $t_{s}=700 \mu \mathrm{s}$. The actual and approximated $\Delta(f)$ have spreads of $0.0100987 \mathrm{MHz}$ and $0.0100985 \mathrm{MHz}$ respectively.

In Figure 10 we see a comparison of the approximated (computed numerically) $\Delta(\omega)$ and the actual $\Delta(\omega)$. Their spreads are similar, meaning that the approximation is valid.

\section{THE NUMERICAL EXTENSION OF THE ANALYSIS}

Simulation and commissioning data are discrete, so the Fourier analysis requires the use of DFTs rather than Fourier transforms. A characteristic of FRS signals obtained from commissioning data is the activation of detectors prior to beam injection, resulting in a period of time with no signal. Analytically, we know that the Fourier transform of the FRS is given by equation (12), suggesting that it is incorrect to take the DFT starting from time bins before $t_{0}$. To understand the properties and correct application of the DFT to the given situation, we created a Toy Model that modeled an emittanceless muon beam with momentum and longitudinal spread hitting a plane-like detector in the ring. For a detector located halfway around the ring, $t_{0}=74.563 \mathrm{~ns}$ for a beam with symmetric longitudinal spread. If the signal is sampled starting at a time $t<t_{0}$ we observe a distorted spectrum. If the signal is sampled starting with a time bin centered at $t_{0}$, the correct spectrum is observed. This fact is demonstrated in Figure 11. 

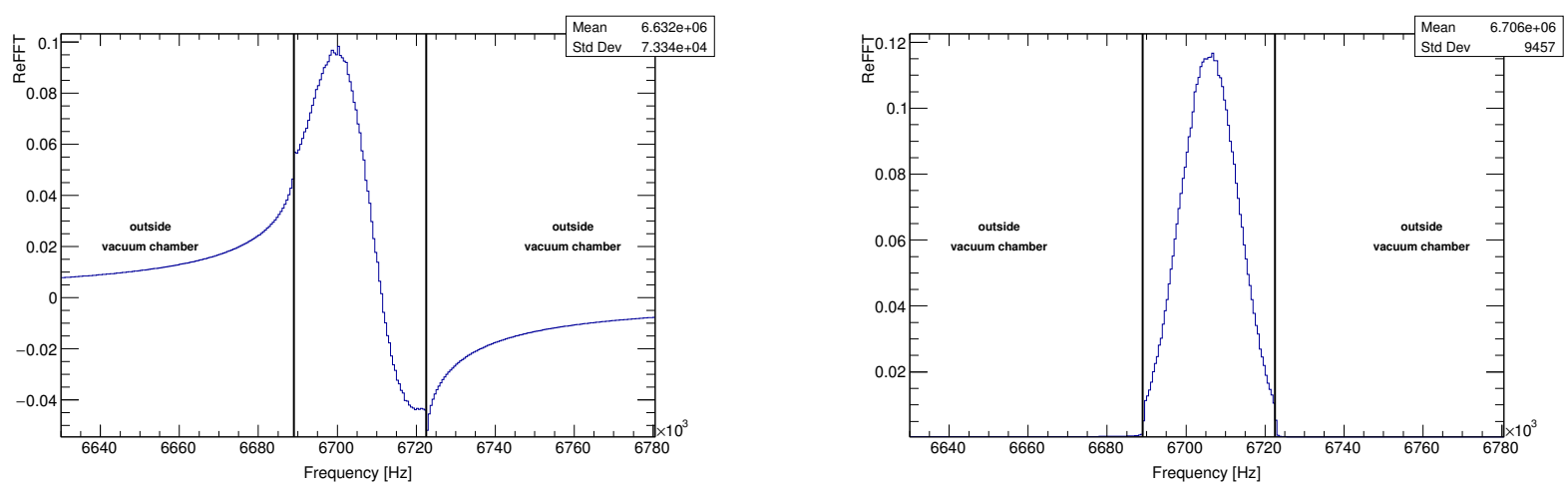

FIG. 11. DFTs of the FRS from Figure 2. The DFT on the right uses $t_{0}=50 \mathrm{~ns}$ and the one the left uses $t_{0}=74.563 \mathrm{~ns}$.

The DFT was accomplished by passing $\mathrm{C}++$ code to the ROOT data analysis framework, which uses the built-in FFTW3 C library to perform the DFT using the fast Fourier transform (FFT) algorithm.

\section{SIMULATION VS. COMMISSIONING DATA}
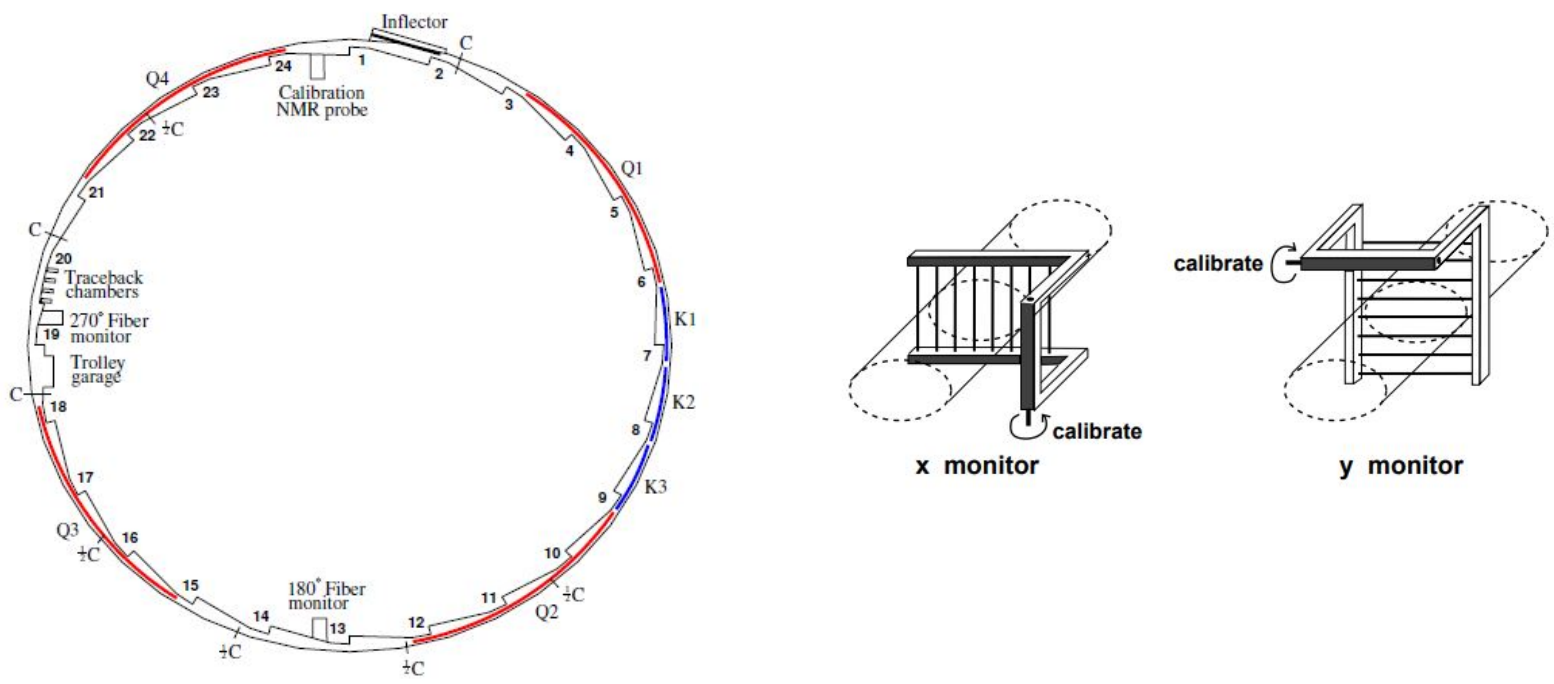

FIG. 12. Left, sketch of the storage ring with locations of the scintillating-fiber beam monitors marked $^{2}$. Right, sketches of the scintillating-fiber beam monitors ${ }^{1}$.

The storage ring features two sets of scintillating-fiber beam monitors located $180^{\circ}$ and $270^{\circ}$ from the injection point (see Figure 12). The monitors measure the beam profile as a function of time, and are able to operate for several tens of microseconds before the beam 
is degraded ${ }^{1}$. To extract the revolution frequency distribution, we used the intensity signal from commissioning run 1835 obtained from fiber beam monitor 2, which measures the beam profile in the radial direction and is located $180^{\circ}$ from the injection point. Due to the delivery ring being shut off, the fill featured the presence of muons, pions, and protons, with the latter being the predominant constituent. Additionally, the lack of Recycler RF cavities prevented the preservation of the intended "W-shape" bunch profile. Furthermore, the intensity signal is not the FRS exactly. Factors like particle decays and particle losses must be factored out from the signal obtained from the beam monitors. Since protons were present in much greater quantities than other particles, no particle decays were factored out. Other effects were not removed due to the need for more studies of their impacts or due to their negligible influence. Due to these imperfections, we expected to extract at most a clear peak centered near the design frequency for protons. Figure 13 features the signal obtained from the commissioning data.

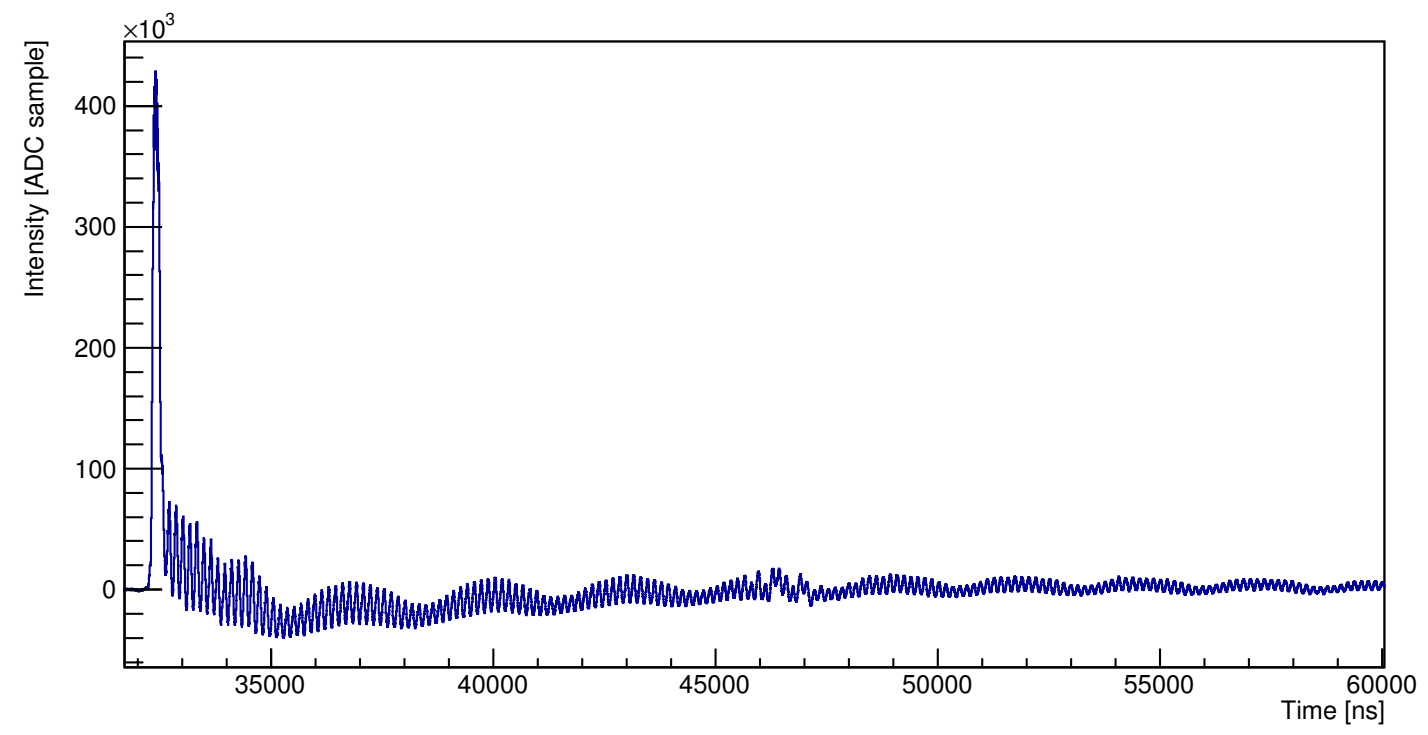

FIG. 13. FRS from the commissioning data. Injection occurs at $\sim 32 \mu \mathrm{s}$, with $t_{0}=32392.5 \mathrm{~ns}$. The noise between $46-47 \mu \mathrm{s}$ is due to a change in ESQ voltage. The baseline undershoot is due to a detector effect. The decrease in amplitude soon after injection is due to the particle losses.

The commissioning data were compared to simulations accomplished using the BMAD subroutine library for relativistic charged-particle dynamics simulations. The simulation matched the commissioning run 1835 as closely as possible, but was unable to model the 
baseline undershoot or the lack of delivery ring and Recycler RF cavities. Due to the predominance of protons in the fill, the simulated beam featured only protons. We see in Figures 14 and 15 the signal obtained from simulation.

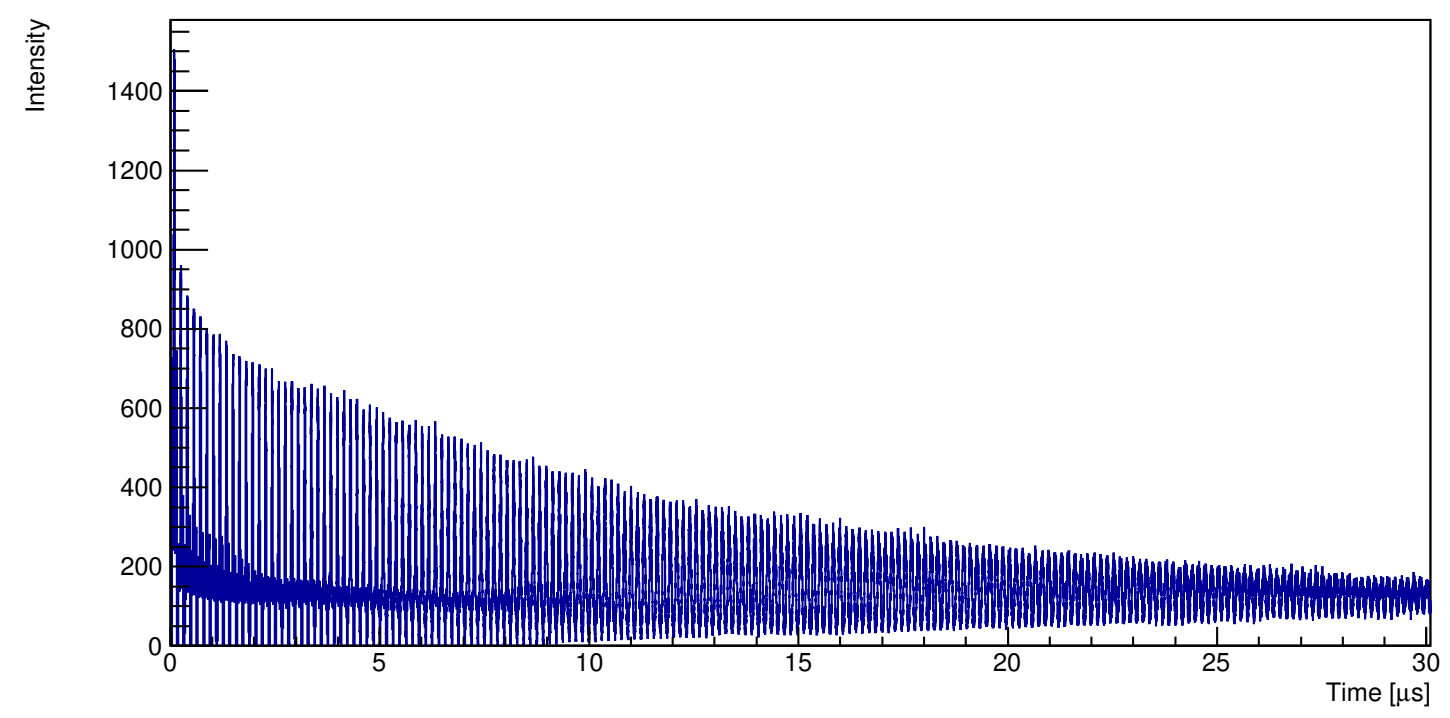

FIG. 14. FRS from the BMAD simulation.

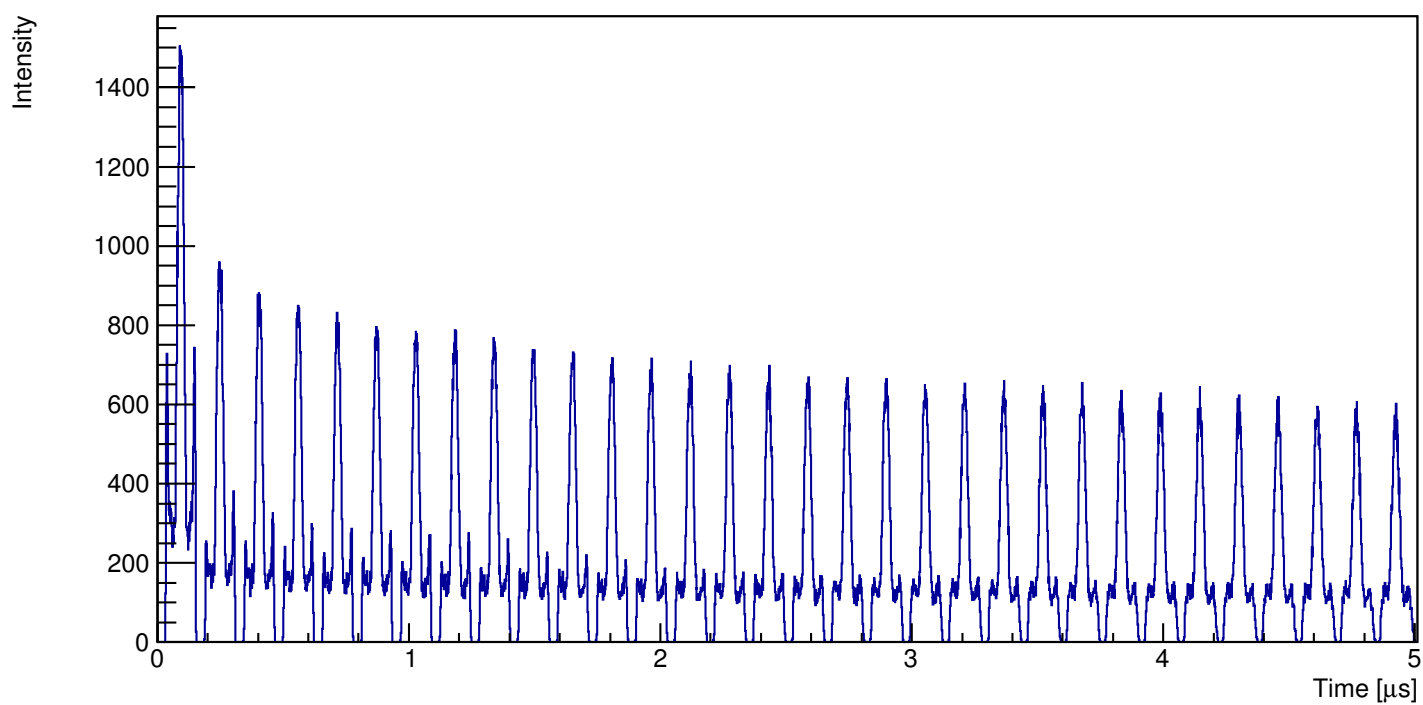

FIG. 15. FRS from the BMAD simulation zoomed in to show the "W-shape" longitudinal bunch structure. $t_{0}=92.5 \mathrm{~ns}$. 

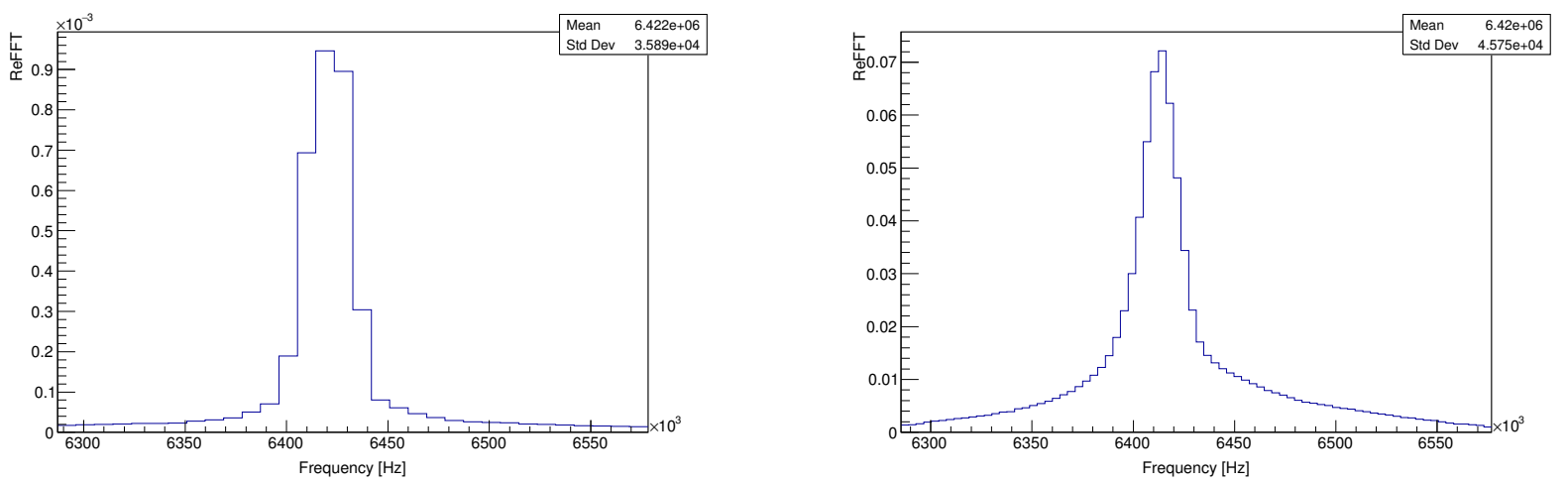

FIG. 16. DFT of FRS from the BMAD simulation. Left, DFT of the FRS from the commissioning run. The peak centers are set apart by $757 \mathrm{kHz}$.

In Figure 16 we see a comparison of the DFT from simulation data to that from the commissioning data. We see clear peaks for both with a difference of $757 \mathrm{kHz}$ between them. The peaks are close to the design frequency of the protons $(6.42 \mathrm{MHz})$.

The proximity of the first harmonics of the DFTs suggest that the developed analysis is correct. However, work remains in understanding what effects various backgrounds, like particle losses, have on the fast rotation signal. In addition, the actual experiment will draw the FRS from calorimeter station data rather than scintillating-fiber beam monitors. The azimuthal width of the stations and their reliance on decay positrons rather than stored muons forces some modifications of the developed analysis.

\section{ACKNOWLEDGMENTS}

Special thanks goes to my mentor Brendan Casey, as well as to Antoine Chapelain and David Rubin for their invaluable thoughts and ideas. This work was supported in part by the U.S. Department of Energy, Office of Science, Office of Workforce Development for Teachers and Scientists (WDTS) under the Science Undergraduate Laboratory Internship (SULI) program.

\section{REFERENCES}

${ }^{1}$ J. P. M. et al, "Muon (g-2): experiment and theory," Rep. Prog. Phys. 70 (2007).

2 J. G. et al, "Muon (g-2) technical design report," (2015), arXiv:1501.06858. 
${ }^{3}$ F. Jegerlehner and A. Nyffeler, "The muon g-2," Phys. Rep. 477, 110 (2009).

${ }^{4}$ Y. Orlov, C. S. Ozben, and Y. K. Semertzidis, "Muon revolution frequency distribution from a partial-time fourier transform of the g-2 signal in the muon g-2 experiment," Nucl. Instrum. Methods Phys. Res. A 482, 9 (2002).

${ }^{5}$ G. B. Arfken and H. J. Weber, "Mathematical methods for physicists," (Elsevier, 2005) Chap. 15, pp. 936-937, 6th ed.

${ }^{6}$ L. Landau and E. Lifschitz, "The classical theory of fields," (Pergamon, New York, 1971) Chap. 7, pp. 143-144, 3rd ed. 